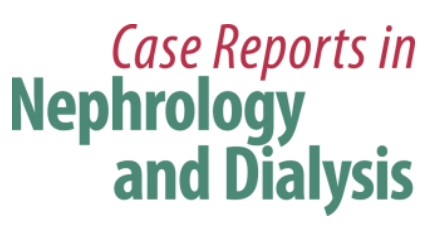

\title{
Glomerulocystic Kidney Disease and Hepatoblastoma in an Infant: A Rare Presentation
}

\author{
Rumina Zaman ${ }^{a} \quad$ Alec Maggi ${ }^{b} \quad$ Sudeep K. Rajpoot ${ }^{c} \quad$ Divya-Devi Joshi $^{a}$ \\ ${ }^{a}$ Miller Children's and Women's Hospital, Long Beach, Calif., and 'Drexler University, \\ Philadelphia, Pa., USA; ${ }^{C}$ American University of Antigua, St. John's, Antigua and Barbuda
}

\section{Key Words}

Hepatoblastoma · Beckwith-Wiedemann syndrome · Glomerulocystic kidney disease · Hepatocyte growth factor $\cdot$ Insulin-like growth factors

\begin{abstract}
Glomerulocystic kidney disease (GCKD) is a rare condition comprising heritable and nonheritable types [Oh et al.: Nephron 1986;43:299-302]. Hepatoblastoma is a sporadically occurring tumor of embryonal origin that is associated with overgrowth syndrome and renal cysts. A concurrent presentation of GCKD with hepatoblastoma was first described in 1989 [Rao et al.: Jpn J Surg 1989;19:583-585]. We report the simultaneous presentation of hepatoblastoma and GCKD in a 5-month-old child and explore the probability of insulin-like growth factors, insulin-like growth factor-binding protein and Beckwith-Wiedemann gene mutation as a putative cause.

(c) 2015 S. Karger AG, Basel
\end{abstract}

\section{Introduction}

Described in 1941, glomerulocystic kidney disease (GCKD) is a rare condition characterized by cortical microcysts in Bowman's spaces [1]. GCKD most commonly presents in newborns and children. It can be sporadic or inheritable, is found in syndromes such as tuberous sclerosis and trisomy 13 and 18 and can affect multiple organs [2]. There is a known association of hypoplastic glomerulocystic disease with mutation of hepatocyte nuclear factor $1 \beta$ (HNF-1 $\beta$ ) [3]. Insulin-like growth factor (IGF) is associated with renal growth and cyst formation. 


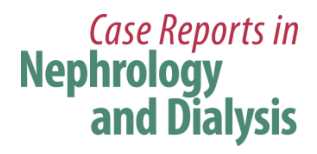

\begin{tabular}{l|l}
\hline Case Rep Nephrol Dial 2015;5:200-203 \\
\hline DOI: 10.1159/000439520 & $\begin{array}{l}\text { C 2015 S. Karger AG, Basel } \\
\text { www.karger.com/cnd }\end{array}$ \\
\hline
\end{tabular}

Zaman et al:: Glomerulocystic Kidney Disease and Hepatoblastoma in an Infant: A Rare Presentation

Hepatoblastoma, a malignant pediatric liver neoplasm of embryonal origin, is associated with Beckwith-Wiedemann syndrome (BWS), Wilms' tumor and renal cysts. Our Medline search found only 6 cases of an association of hepatoblastoma with GCKD.

\section{Case Report}

A 2-month-old, 34-week-gestational-age, African-American female presented to the emergency department with respiratory distress, abdominal distention and a 4-day history of nonbloody, yellow, watery stool. She was admitted to the hospital for respiratory distress.

The infant had been born via normal vaginal delivery without any prenatal problems. After birth, she had spent 10 days in the neonatal intensive care unit for respiratory distress and suspected sepsis. She had been intubated at that time and received antibiotics for a positive blood culture. She had subsequently been discharged in a stable condition. Her developmental course since then had been normal. Both parents and 2 siblings (a brother and sister) were healthy. The family history was noncontributory for cancer or renal or liver disease.

Upon admission, the oncology service was consulted for abdominal distention. An abdominal ultrasound showed a liver mass, and both kidneys were found to be highly echogenic. The right kidney measured $10.3 \mathrm{~cm}$ and the left kidney $9.9 \mathrm{~cm}$, i.e. they were both enlarged for the infant's age. Due to the hyperechoic large kidneys, the patient was suspected of having renal cystic disease. A computed tomography scan showed pulmonary metastases.

At the time of admission, the patient's hemogram (complete blood count) and comprehensive metabolic panel revealed normocytic anemia, mildly elevated liver enzymes, serum blood urea nitrogen of $20 \mathrm{mg} / \mathrm{dl}$ and creatinine of $0.5 \mathrm{mg} / \mathrm{dl}$. The laboratory values included $\alpha$-fetoprotein of 1,206,438 ng/ml (normal 0-8 ng/ml), IGF of $100 \mathrm{ng} / \mathrm{ml}$ (normal 18-146 $\mathrm{ng} / \mathrm{ml}$ ) and IGF-binding protein 3 of 2,625 ng/ml (normal 1,039-3,169 ng/ml).

Two days after admission, she developed hypertension and decreased urine output. Ultrasound-guided liver and kidney biopsies showed hepatoblastoma and GCKD, respectively. A bone marrow biopsy revealed no evidence of metastatic tumor, and her chromosome analysis showed a normal female karyotype with no evidence of clinically significant numerical or structural chromosome abnormalities.

Given the significant association with hepatic tumor and renal cystic diseases, we also performed genetic testing for BWS: chromosomal rearrangement, methylation of imprinting control regions 1 and 2, and sequence analysis of cyclin-dependent kinase inhibitor $1 \mathrm{C}$. The results of these studies were normal, ruling out an association for our patient [4].

She was diagnosed with stage 4 hepatoblastoma and was treated with cisplatin, vincristine and fluorouracil. Despite appropriate hydration, following the first chemotherapy cycle, the patient's renal function deteriorated, and she developed anuric renal failure with serum BUN of $54 \mathrm{~g} / \mathrm{dl}$ and serum creatinine of $5.3 \mathrm{mg} / \mathrm{dl}$. She also developed pulmonary edema requiring intubation and dialysis. Based on her clinical condition, the decision was made to not give fluorouracil. Despite all supportive measures, she remained anuric, and no further chemotherapy or surgical intervention was attempted. Echocardiography showed mild left ventricular hypertrophy.

The patient remained in critical condition, requiring ventilator and inotrope support. She developed disseminated intravascular coagulation requiring multiple transfusions of packed red blood cells, platelets and fresh frozen plasma. She remained anuric and hemodialysis dependent. Despite intensive care treatment for 40 days, the patient died of cardiopulmonary failure. The autopsy revealed pulmonary congestion and edema with bilateral 


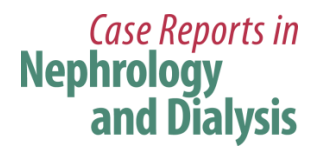

\begin{tabular}{l|l}
\hline Case Rep Nephrol Dial 2015;5:200-203 \\
\hline DOI: $10.1159 / 000439520$ & $\begin{array}{l}\text { C 2015 S. Karger AG, Basel } \\
\text { www.karger.com/cnd }\end{array}$ \\
\hline
\end{tabular}

Zaman et al.: Glomerulocystic Kidney Disease and Hepatoblastoma in an Infant: A Rare Presentation

bronchopneumonia, splenomegaly, hepatomegaly with a right lobe hepatoblastoma of $7 \times 4$ $\times 5 \mathrm{~cm}$, common bile duct obstruction and right axillary lymphadenopathy. Both kidneys were enlarged, with bilateral glomerulocystic disease and pulmonary metastases. There was generalized edema with pleural effusion, pericardial effusion and ascites.

The medical team's recommendation to the parents and siblings to obtain a renal ultrasound, urinalysis, hemogram and electrolyte panel was declined.

\section{Discussion}

GCKD and hepatoblastoma typically present in infants and young children. Only a few case reports have described the presence of both diseases in a patient $[5,6]$. GCKD can be divided into three categories: (1) GCKD comprising nonsyndromal inheritable and sporadic forms of severely cystic kidneys in children and adults, (2) glomerulocystic kidneys associated with inheritable malformation syndromes and (3) glomerular cysts in dysplastic kidneys. Many patients have a family history of autosomal dominant polycystic kidney disease [7].

Although it is the most common primary liver tumor in children, hepatoblastoma is an uncommon malignancy. It mostly occurs in infants 2 months to 3 years of age and is associated with very high levels of $\alpha$-fetoprotein. It originates from embryonal liver precursor cells and typically presents as an asymptomatic abdominal mass with nausea, anorexia or vomiting. It is associated with overgrowth syndromes such as BWS, which is characterized by large birth weight, organomegaly and macroglossia. Hepatoblastoma can also be associated with familial adenomatous polyposis coli and various diseases of the kidneys such as horseshoe kidneys.

There are interesting reports describing the coexistence of GCKD and hepatoblastoma. Rao et al. [5] first proposed the association in 1989. Both conditions arise in infancy, and IGFs play an important role in the development of both the liver and kidneys. Alterations in the growth-regulatory gene (IGF-2), on chromosome 11p15, occur in patients with hepatoblastoma [8]. During early liver development, these patients have increased IGF-2 expression, which subsequently changes to IGF-1. During glomerular development, both IGF-1 and IGF-1R are expressed, their patterns of expression being disrupted in animal models and in human examples of renal disease. Indeed, IGF has been shown to play a role in signaling to maintain glomerular integrity via the preservation from damage of podocytes and the glomerular basement membrane [9].

Alterations in growth-regulatory genes on chromosome 11p15.5 are found in BWS, which has a higher incidence of hepatoblastoma. Genetic testing in our patient was not consistent with a diagnosis of BWS. The levels of IGF-1 and IGF-binding protein in our patient were normal. Unfortunately, we did not obtain IGF-2 levels [8].

A transcription factor mutation of the growth factor HNF-1 $\beta$ is associated with various renal cystic diseases including GCKD [10]. The hypothesis that mutation of HNF-1 might be associated with liver tumors as well as renal cystic disease has, however, been disproved [11].

\section{Conclusions}

Our case is a rare presentation of both glomerulocystic disease and hepatoblastoma in an infant. One may dismiss the association as being merely coincidental. It is, however, intriguing that all reported cases have an identical clinical presentation in infancy, that both 
Zaman et al:: Glomerulocystic Kidney Disease and Hepatoblastoma in an Infant: A Rare Presentation

GCKD and hepatoblastoma arise in infancy, and that both are affected by IGF in their development. Mutations in the growth-regulatory gene are found in hepatoblastoma, and IGF plays a role in glomerular integrity.

It is, therefore, conceivable that these two diseases are linked. Early recognition could have significant clinical consequences. It is important for clinicians to rule out a diagnosis of hepatoblastoma in the presence of GCKD, and vice versa, in an infant.

\section{Statement of Ethics}

The authors have no ethical conflicts to disclose.

\section{Disclosure Statement}

The authors declare no conflicts of interest.

\section{References}

$\longrightarrow 1$ Oh Y, Onoyama K, Kobayashi K, et al: Glomerulocystic kidneys. Report of an adult case. Nephron 1986;43:299-302.

-2 Lennerz JK, Spence DC, Iskandar SS, Dehner LP, Liapis H: Glomerulocystic kidney: one hundred-year perspective. Arch Pathol Lab Med 2010;134:583-605.

-3 Mache CJ, Preisegger KH, Kopp S, Ratschek M, Ring E: De novo HNF-1 $\beta$ gene mutation in familial hypoplastic glomerulocystic kidney disease. Pediatr Nephrol 2003;17:1021-1026.

4 Prawitt D, Enklaar T, Gärtner-Rupprecht B, et al: Microdeletion of target sites for insulator protein CTCF in a chromosome 11p15 imprinting center in Beckwith-Wiedemann syndrome and Wilms' tumor. Proc Natl Acad Sci USA 2005;102:4085-4090.

-5 Rao PS, Krishna A, Rohatgi M: Multicystic kidney in association with hepatoblastoma - a case report. Jpn J Surg 1989;19:583-585.

6 Bhaskar KV, Joshi K, Banerjee CK: Hepatoblastoma with glomerulocystic disease - a mere coincidence or an association? Nephron 1990;54:273-274.

7 Bernstein J: Glomerulocystic kidney disease - nosological considerations. Pediatr Nephrol 1993;7:464-470.

8 Gray SG, Erikkson T, Ekström C, Holm S, von Schweinitz D, Kogner P, Sandstedt B, Pietsch T, Ekstöm TJ: Altered expression of members of the IGF-axis in hepatoblastoma. Br J Cancer 2000;82:1561-1567.

-9 Bridgewater DJ, Dionne JM, Butt MJ, Pin CL, Matsell DG: The role of the type I insulin-like growth factor receptor (IGF-IR) in glomerular integrity. Growth Horm IGF Res 2008;18:26-37.

10 Igarashi P, Shao X, McNally BT, Hiesberger T: Roles of HNF-1 $\beta$ in kidney development and congenital cystic diseases. Kidney Int 2005;68:1944-1947.

11 Abdul-Rahman OA, Edghill EL, Kwan A, Enns GM, Hattersley AT: Hypoplastic glomerulocystic kidney disease and hepatoblastoma: a potential association not caused by mutations in hepatocyte nuclear factor $1 \beta$. J Pediatr Hematol Oncol 2009;31:527-552. 